\title{
Measurements of stress-optic coefficient in polymer optical fibers
}

\author{
Marcin K. Szczurowski ${ }^{1}$, Tadeusz Martynkien ${ }^{1}$, Gabriela Statkiewicz-Barabach ${ }^{1}$, \\ Waclaw Urbanczyk ${ }^{1, *}$, Lutful Khan ${ }^{2}$, David J. Webb ${ }^{2}$ \\ ${ }^{1}$ Institute of Physics, Wroclaw University of Technology, Wybrzeze Wyspianskiego \\ 27, 50-370 Wroclaw, Poland \\ ${ }^{2}$ Photonics Research Group, Aston University, Birmingham, B4 7ET, UK
}

\begin{abstract}
We have systematically measured the differential stress-optic coefficient, $\Delta \mathrm{C}$, in a number of PMMA fibers drawn with different stress, ranging from 2 up to $27 \mathrm{MPa} . \Delta \mathrm{C}$ was determined in transverse illumination by measuring the dependence of birefringence on additional axial stress applied to the fiber. Our results show that $\Delta \mathrm{C}$ in PMMA fibers has a negative sign and ranges from -4.5 to $-1.5 \times 10^{-12} \mathrm{~Pa}^{-1}$ depending on the drawing stress. Increase of the drawing stress results in greater initial fiber birefringence and lower $\Delta \mathrm{C}$.
\end{abstract}

OCIS codes: $060.2400,060.2300,060.2290$

Development of polymer optical fibers has opened new application opportunities in optical communication [1] and sensing [2] because of their low cost, easy handling and very good flexibility. Optical anisotropy in polymers of different types has been investigated for many years due to their potential use in various photonic devices $[3,4]$. Although PMMA is a very popular polymer widely used for industry, there are many inconsistencies in the literature concerning its differential stressoptic coefficient $\Delta \mathrm{C}=\mathrm{C}_{2}-\mathrm{C}_{1}$. Measurements performed in PMMA discs $(18 \mathrm{~mm}$ diameter, $10 \mathrm{~mm}$ thick) showed $\Delta \mathrm{C}$ equal to $-3.3 \times 10^{-12} \mathrm{~Pa}^{-1}[4]$, but in [5] the measurements carried out in discs of different size (13.03 mm diameter, $2.85 \mathrm{~mm}$ thick) gave a value of $\Delta \mathrm{C}=5.5 \times 10^{-10} \mathrm{~Pa}^{-1}$. Another quite different result was reported in [6], $\Delta \mathrm{C}=-1.08 \times 10^{-10} \mathrm{~Pa}^{-1}$. Very systematic studies of the optical and mechanical properties of bulk PMMA (Plexiglas 55) are presented in [7]. In particular, the strain-optic 
coefficients for this material have been measured $\mathrm{p}_{11}=0.300, \mathrm{p}_{12}=0.297$, which lead to a calculated value of $\Delta \mathrm{C}=-2.4 \times 10^{-12} \mathrm{~Pa}^{-1}$, assuming the following material constants: refractive index $\mathrm{n}=1.4928$ at $\lambda=656 \mathrm{~nm}$ [7], Young's modulus $\mathrm{E}=2.8 \pm 0.2 \mathrm{GPa}$ [8], and Poisson's ratio $v=0.345$ [9].

The goal of this work was to systematically measure the coefficient $\Delta \mathrm{C}=\mathrm{C}_{2}-\mathrm{C}_{1}$ in a number of PMMA fibers drawn with different stress and to investigate the effect of temperature annealing on this parameter. To determine $\Delta \mathrm{C}$, we measured the dependence of fiber birefringence upon additional axial stress $\sigma_{\mathrm{z}}^{\text {applied }}$ applied to the fiber. The drawing stress and geometrical parameters of the examined fibers are gathered in Table 1. The fibers used in this study were all drawn from homogeneous, nominally pure PMMA rods obtained from AMARI plastics. The mean molecular weight was determined by gas permeation chromatography to be 87000 . To prevent the preforms from bubbling in the draw tower oven, they were annealed at $80^{\circ} \mathrm{C}$ for 48 hours immediately prior to fiber drawing. The fibers were all drawn with a hot-zone temperature of $181 \pm 2^{\circ} \mathrm{C}$ and the fiber tension and consequently the drawing stress controlled by adjusting the preform feed and fiber take-up rates. A selection of the fiber samples was annealed at $100^{\circ} \mathrm{C}$ for 20 hours.

To measure the fiber birefringence in the direction perpendicular to the symmetry axis, we used a system employing a Senarmont compensation principle shown in Fig.1. The measured retardation distribution $\mathrm{R}(\mathrm{x})=\Sigma_{\mathrm{x}}(\mathrm{x})-\Sigma_{\mathrm{z}}(\mathrm{x})$ is related to the axial stress distribution $\sigma_{\mathrm{z}}(\mathrm{r})$ in the fibers cross-section by the inverse Abel transformation [10]:

$$
\sigma_{z}(r) \Delta C=\frac{-1}{\pi} \int_{r}^{r_{0}} \frac{d R(x) / d x}{\sqrt{x^{2}-r^{2}}} d x
$$

where $\mathrm{r}_{0}$ is the fiber radius, $\sigma_{z}(r)$ is the axial stress, and $\mathrm{dR} / \mathrm{dx}$ is a derivative of measured retardation. The stress optic coefficient $\Delta C$ can be easily retrieved by applying increasing axial load to the fiber and finally by plotting the dependence $\Delta C \sigma_{z}^{\text {calculated }}$ versus $\sigma_{z}^{\text {applied }}$ with $\Delta C$ being a proportionality coefficient. Due to cylindrical symmetry, stress induced optical anisotropy of the fiber is characterized by three principal indices $n_{z}, n_{r}, n_{\theta}$. When axial stress is applied to a fiber made of uniform material, radial and tangential stress components in the fiber cross section are significantly lower than $\sigma_{z}$ $\left(\sigma_{z}>>\sigma_{r}, \sigma_{z}>>\sigma_{\Theta}\right)$, which results in: 


$$
n_{z}=n+C_{1} \sigma_{z}, \quad n_{\Theta}=n+C_{2} \sigma_{z}, \quad n_{r}=n+C_{2} \sigma_{z}
$$

and finally

$$
n_{x}-n_{z}=\Delta C \sigma_{z}
$$

where $\Delta \mathrm{C}=\mathrm{C}_{2}-\mathrm{C}_{1}$ is the differential stress-optic coefficient and $n_{x}=n_{\theta}=n_{r}$. As the investigated fibers are made of uniform PMMA, the inverse Abel transformation of the measured retardation distribution $\mathrm{R}(\mathrm{x})$ provides directly information about the birefringence $n_{x}-n_{z}$ induced by applied axial stress.

We first measured $\Delta C$ in a standard SMF-28 silica fiber, which was used as a reference to test our measurement and processing method. Silica is a very well characterized material, thus allowing us to compare the results obtained using our method with a reliable value obtained from the literature [11], $\Delta \mathrm{C}=(3.57 \pm 0.03) \times 10^{-12} \mathrm{~Pa}^{-1}$ at $526 \mathrm{~nm}$. By the linear fitting of measured fiber birefringence in the pure silica cladding versus applied stress, we obtained a value for the stress-optic coefficient of $\Delta \mathrm{C}=(3.52 \pm 0.01) \times 10^{-12} \mathrm{~Pa}^{-1}$. The measured value is not significantly different from the one reported in the literature, which gives confidence in our measurement and processing methods.

Similar measurements were performed for a series of PMMA fibers drawn with different stress (Table 1). In Fig.2 we show the birefringence distribution reconstructed from the retardation measured for different axial stress $\sigma_{z}^{\text {applied }}$ applied to fiber $1 \mathrm{~b}$, which was drawn using a relatively small drawing stress of 1.86 MPa. For all investigated fibers, the applied axial stress was gradually increased until the fiber broke. As it can be clearly seen in Fig.2, the reconstructed distribution of stress-induced birefringence $\Delta C \sigma_{z}$ is not perfectly uniform in the fiber's cross-section. An increase of $\Delta C \sigma_{z}(\mathrm{r})$ near the fiber edges by about $10 \%$ is most probably caused by the higher cooling rate of the external layers of the fiber during the drawing process. To determine $\Delta C$ in the polymer fibers, we averaged the measured distribution of $\Delta C \sigma_{z}(\mathrm{r})$ in the fiber cross-section and plotted the dependence of the averaged $\sigma_{z} \Delta C=n_{x}-n_{z}$ as a function of applied stress, see Fig.3. In order not to obscure this figure, we show only the results obtained for a few fibers having significantly different $\Delta C$. The birefringence induced by applied axial stress increases linearly for small values of $\sigma_{z}^{\text {applied }}$. The stress optic coefficient $\Delta C$ was determined for each PMMA fiber by linear approximation of the dependence of $\Delta C \sigma_{z}^{\text {calculated }}$ 
(averaged over the fiber cross-section) versus $\sigma_{z}^{\text {applied }}$ for small values of $\sigma_{z}^{\text {applied }}$, with $\Delta C$ being a regression coefficient. The dependence of the stress-optic coefficient $\Delta C$ upon drawing stress is presented in Fig.4. The value of $\Delta C$ increases against $\sigma_{\text {drawing }}$ from -4.5 to $-1.5 \times 10^{-12} \mathrm{~Pa}^{-1}$ in a nonlinear manner and appears to gradually saturate for high drawing stress. The same measurements were repeated for the fibers annealed at a temperature of $100^{\circ} \mathrm{C}$ for 20 hours. It is interesting that fiber annealing does not change the general trend in the dependence of $\Delta C$ versus $\sigma_{\text {drawing }}$, however the saturation effect for annealed fibers occurs at a greater absolute value of $\Delta C$.

Finally, we show in Fig.5 the dependence of $\Delta \mathrm{C}$ upon residual fiber birefringence, which is clearly linear with no significant impact of the annealing process. This experimental result shows that the absolute value of the stress optic coefficient $\Delta \mathrm{C}$ decreases in the PMMA fibers having greater initial birefringence, which directly corresponds to a greater initial ordering of molecules. As the trend line shown in Fig.5 takes a value of $-4.8 \times 10^{-12} \mathrm{~Pa}^{-1}$ at zero birefringence, we can estimate $\Delta \mathrm{C}$ for the bulk PMMA used to draw the fibers, $\Delta \mathrm{C}=-4.8 \times 10^{-12} \mathrm{~Pa}^{-1}$.

From the series of experiments reported in this letter we conclude that the stress-optic coefficient $\Delta \mathrm{C}$ in PMMA fibers has negative sign and ranges from -4.5 to $-1.5 \times 10^{-12} \mathrm{~Pa}^{-1}$ depending on the drawing stress The dependence of $\Delta \mathrm{C}$ on drawing stress is nonlinear and gradually saturates at greater $\sigma_{\text {drawing. }}$ Annealing has a significant impact on the dependence of the differential stress-optic coefficient on $\sigma_{\text {drawing. }}$. Moreover, our measurements demonstrate that $\Delta \mathrm{C}$ is linearly proportional to initial fiber birefringence with annealing having no impact on the slope of this dependence.

\section{Acknowledgements}

The work described in this paper was partially supported by the PHOSFOS-project ("Photonic Skins For Optical Sensing") funded by the European Commission through the 7th ICT-Framework Programme and the Statutory Grant at Wroclaw University of Technology. M. K. Szczurowski and W. Urbanczyk acknowledge support of the FNP Program "MISTRZ". G. Statkiewicz-Barabach acknowledges support of the FNP Program "START" and "MISTRZ", and the fellowship co-financed by European Social Fund. 


\section{References}

1. Y. Koike, T. Ishigure, E. Nihei, "High-bandwidth graded-index polymer optical fiber," J. Lightwave Technol. 13, 1475-1489 (1995).

2. S. Kiesel, K. Peters, T. Hassan, M. Kowalsky, "Behaviour of intrinsic polymer optical fibre sensor for large-strain applications,” Meas. Sci. Technol. 18, 3144-3154 (2007).

3. M. F. Hossain, H. P. Chan, M. A. Uddin, "Simultaneous measurement of thermo-optic and stressoptic coefficients of polymer thin films using prism coupler technique,” Appl. Opt. 49, 403-410 (2010).

4. H. Ohkita, K. Ishibashi, D. Tsurumoto, A. Tagaya, Y. Koike, "Compensation of the photoelastic birefringence of a polymer by doping with an anisotropic molecule," Applied Physics A: Materials Science and Processing 81, 617 - 620 (2005).

5. T. Koyama, Y. Zhu, T. Otsuka, T. Takada, Y. Murooka, “An automatic measurement system for 2-dimensional birefringence vector distribution," 6th International Conference on Conduction and Breakdown in Solid Dielectrics, Västeräs, Sweden, Proceedings of IEEE, 557 - 560 (1998).

6. W. Xu, X. F. Yao, H. Y. Yeh, G. C. Jin, "Fracture investigation of PMMA specimen using coherent gradient sensing (CGS) technology," Polymer Testing 24, 900 - 908 (2005).

7. R. M. Waxler, D. Horowitz A. Feldman, “Optical and physical parameters of Plexiglas 55 and Lexan,” Appl. Opt. 18, 101-104 (1979).

8. M. Silva-López, A. Fender, W. N. MacPherson, J. S. Barton, J. D. C. Jones, D.Zhao, H. Dobb, D. J. Webb, L. Zhang, I. Bennion, "Strain and temperature sensitivity of a single-mode polymer optical fiber,” Opt. Lett. 30, 3129-3131 (2005).

9. D. D. Raftopoulos, D. Karapanos, P. S. Theocaris, "Static and dynamic mechanical and optical behaviour of high polymers," J. Phys. D: Appl. Phys. 9, 869-876 (1976).

10. W. Urbanczyk, K. Pietraszkiewicz, "Measurements of stress anisotropy in fiber preform: modification of the dynamic spatial filtering technique," Appl. Opt. 27, 4117-4122 (1988).

11. W. Primak, D. Post, "Photoelastic constants of vitreous silica and its elastic coefficient of refractive index," J. of Appl. Physics 30, 779-788 (1958). 
Tab.1. Parameters of the investigated fibers. All annealed fibers are indicated by an apostrophe.

Annealing temperature was $100^{\circ} \mathrm{C}$ and duration $20 \mathrm{~h}$.

\begin{tabular}{|c|l|c|c|c|}
\hline $\begin{array}{c}\text { Fiber } \\
\text { sample }\end{array}$ & $\begin{array}{c}\text { Measured } \\
\text { diameter } \\
{[\mathrm{mm}]}\end{array}$ & $\begin{array}{c}\sigma_{\text {drawing }} \\
{[\mathrm{MPa}]}\end{array}$ & $\begin{array}{c}\text { Measured } \Delta \mathrm{C} \\
{\left[\mathrm{Pa}^{-1}\right]}\end{array}$ & $\begin{array}{c}\text { Measured initial } \\
\text { birefringence }\end{array}$ \\
\hline $1 \mathrm{a}^{\prime}$ & 0.605 & 1.98 & $-4.3 \times 10^{-12}$ & $-1.05 \times 10^{-4}$ \\
\hline $1 \mathrm{a}$ & 0.556 & 2.34 & $-4.37 \times 10^{-12}$ & $-1.44 \times 10^{-4}$ \\
\hline $1 \mathrm{~b}^{\prime}$ & 0.329 & 8.86 & $-3.45 \times 10^{-12}$ & $-3.71 \times 10^{-4}$ \\
\hline $1 \mathrm{~b}$ & 0.387 & 5.85 & $-3.62 \times 10^{-12}$ & $-3.23 \times 10^{-4}$ \\
\hline $1 \mathrm{c}^{\prime}$ & 0.126 & 21.3 & $-2.78 \times 10^{-12}$ & $-5.61 \times 10^{-4}$ \\
\hline $1 \mathrm{c}$ & 0.105 & 27.2 & $-1.54 \times 10^{-12}$ & $-7.91 \times 10^{-4}$ \\
\hline $1 \mathrm{~d}^{\prime}$ & 0.396 & 11.4 & $-3.20 \times 10^{-12}$ & $-4.19 \times 10^{-4}$ \\
\hline $1 \mathrm{~d}$ & 0.294 & 18.7 & $-1.79 \times 10^{-12}$ & $-7.91 \times 10^{-4}$ \\
\hline $1 \mathrm{e}^{\prime}$ & 0.291 & 21.2 & $-3.16 \times 10^{-12}$ & $-5.27 \times 10^{-4}$ \\
\hline $1 \mathrm{e}$ & 0.263 & 23.1 & $-1.78 \times 10^{-12}$ & $-8.18 \times 10^{-4}$ \\
\hline
\end{tabular}




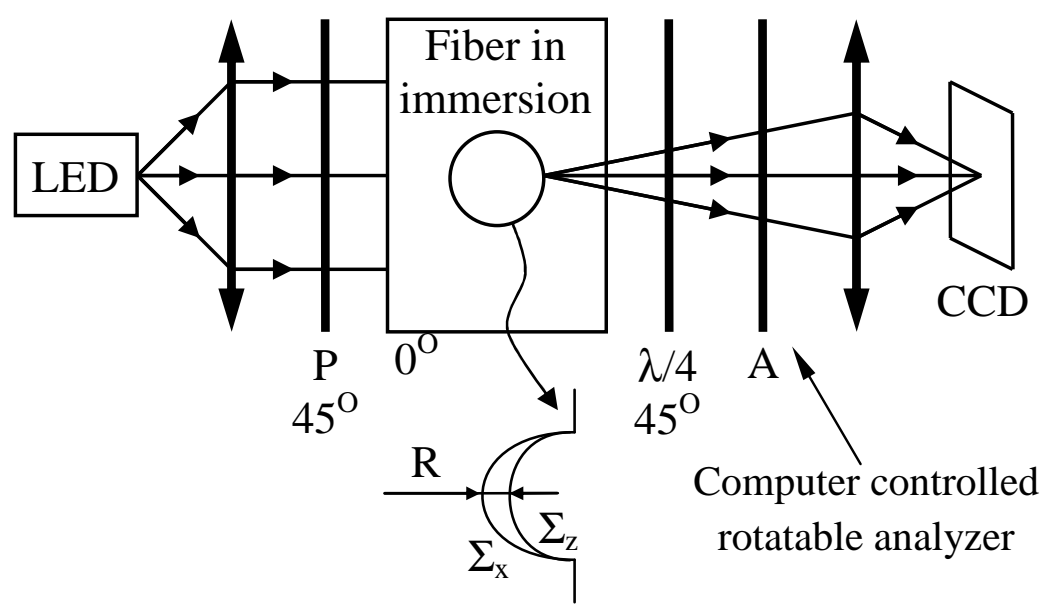

Fig.1. Experimental set-up for measuring retardation distribution, $\lambda=526 \mathrm{~nm}$ 


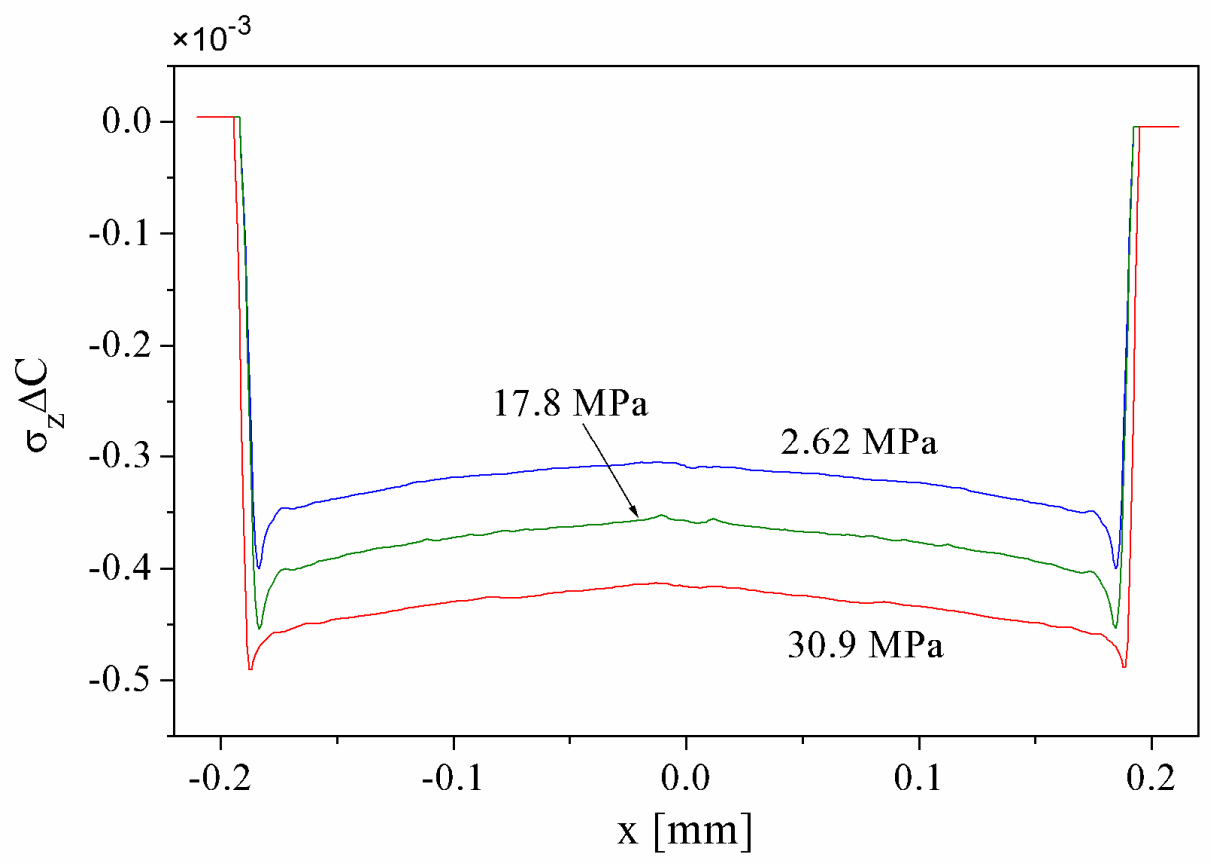

Fig. 2. Distribution of birefringence $\sigma_{z} \Delta C=n_{x}-n_{z}$. reconstructed from measured retardation for different values of applied axial stress $\sigma_{z}^{\text {applied }}$ in the PMMA fiber indicated as $1 b$. 


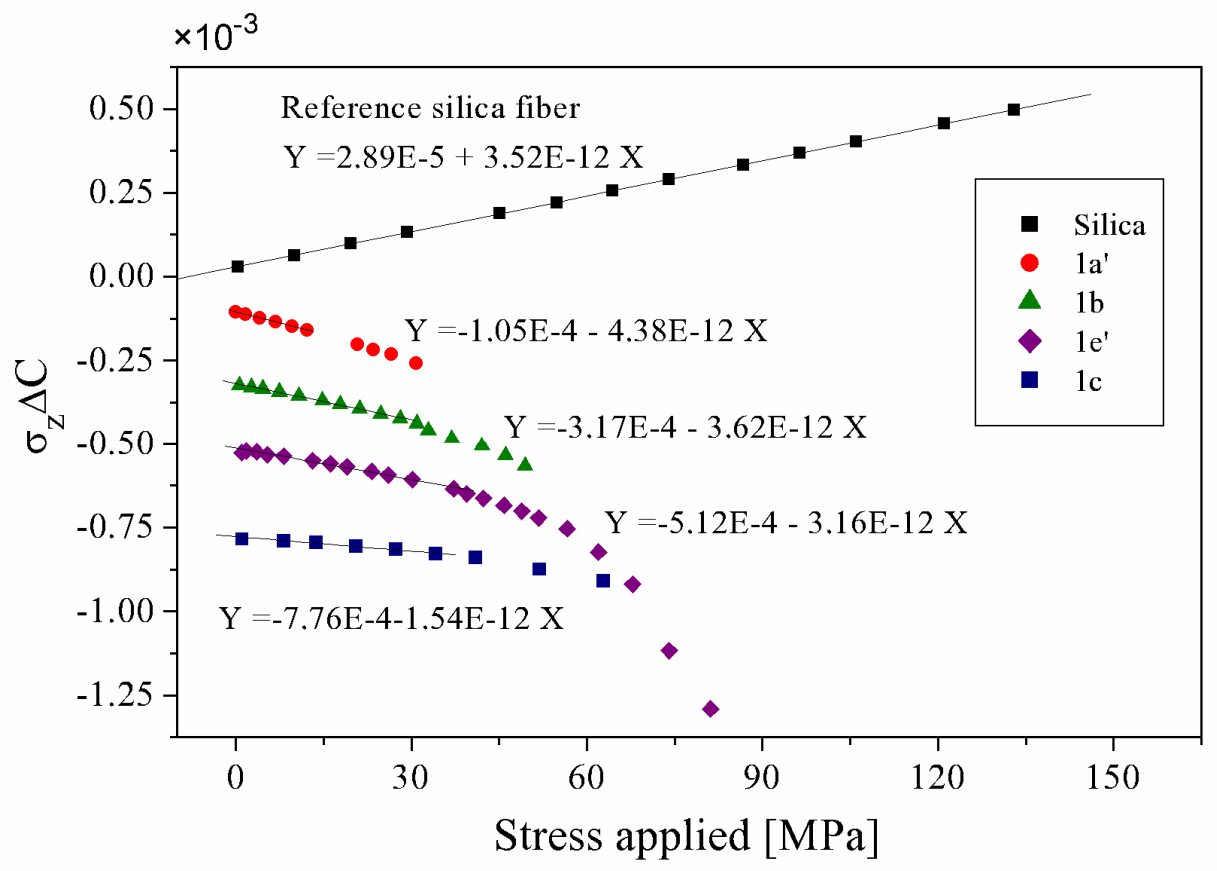

Fig. 3. Dependence of birefringence $\sigma_{z} \Delta C=n_{x}-n_{z}$. in selected PMMA fibers versus applied axial stress. The stress-optic coefficients $\Delta \mathrm{C}$ were obtained by fitting to the linear part of the measured characteristics. 


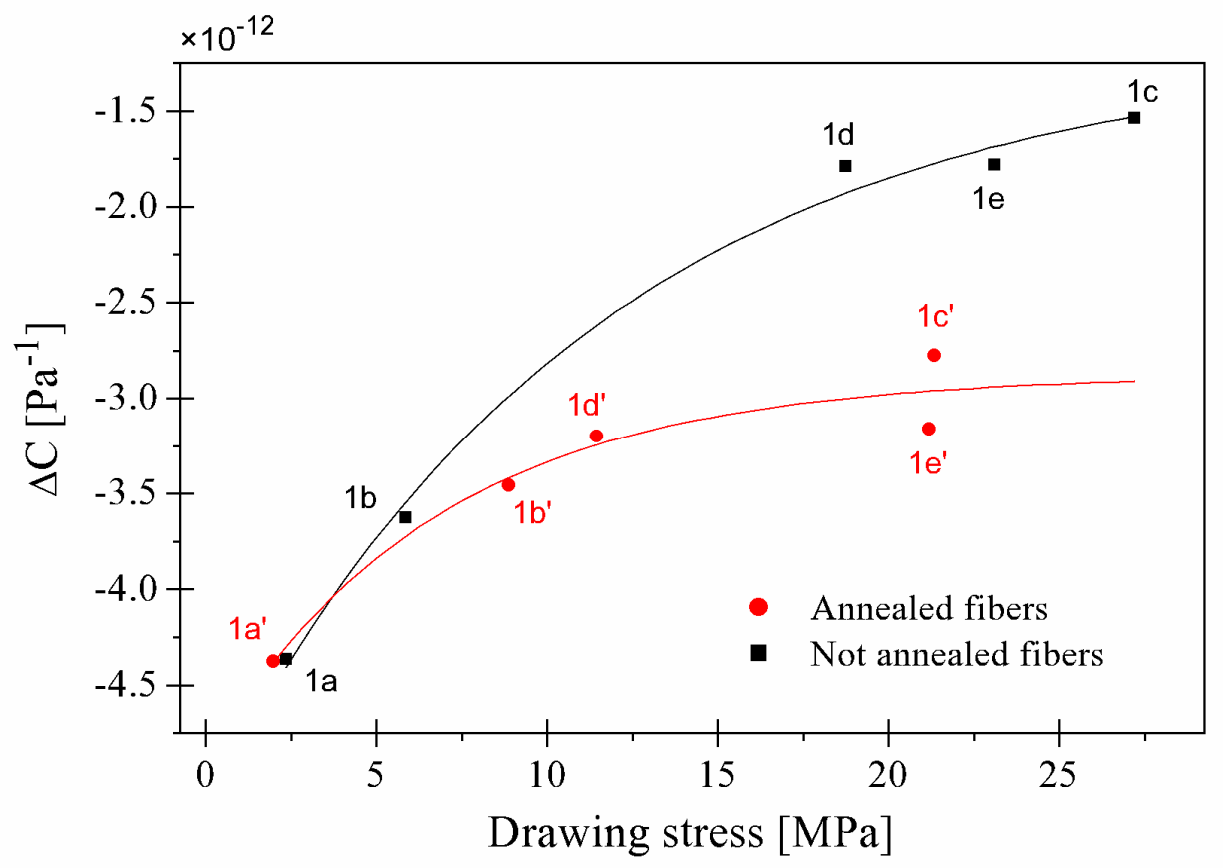

Fig. 4. Dependence of the stress-optic coefficient upon drawing stress in the PMMA fibers. Measurements before and after annealing were carried out for fibers with different cross-sections and as a result the drawing stress for corresponding fibers is not the same. 


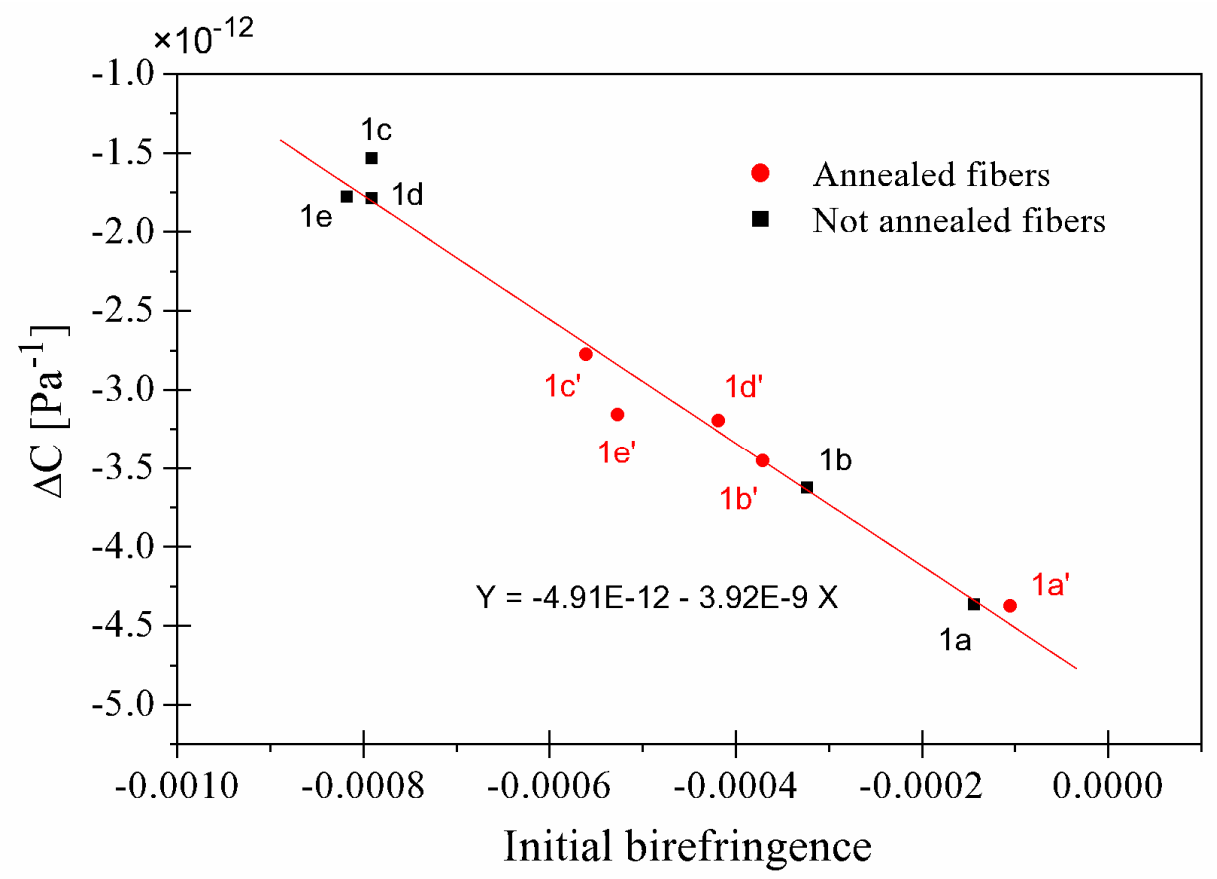

Fig.5. Stress optic coefficient as a function of initial fiber birefringence for the PMMA fibers from Table 1. Measurements before and after annealing were carried out for fibers with different crosssections and as a result the drawing stress for corresponding fibers is not the same. 